\title{
Relative sample control: A quantitative procedure for assessing transfer effects in conditional discrimination problems
}

\author{
JO W. TOMBAUGH and T. N. TOMBAUGH \\ Carleton University, Ottawa, Ontario KIS 5B6, Canada \\ and \\ ANGELO SANTI \\ Wilfrid Laurier University, Waterloo, Ontario N2L 3C5, Canada
}

\begin{abstract}
A three-key paradigm was used to compare acquisition and transfer performance on two conditional discrimination problems: matching and oddity. After a stable level of performance was achieved on the initial task, a novel transfer stimulus was administered. Equivalent amounts of transfer were observed for both matching and oddity when the data were analyzed by a measure (relative sample control) that reflected the relative degree to which the sample stimulus controlled behavior. The position is advanced that these data can be interpreted directly on the basis of well-known transfer parameters (e.g., stimulus similarity and response compatibility) without requiring any special assumptions concerning the coding of the sample stimulus. Additionally, the type of data analysis employed was found to be a useful technique for evaluating conditional discrimination results when possible response biases are present.
\end{abstract}

The assessment of transfer effects in conditional discrimination experiments is critically dependent upon the selection of baseline performance estimates against which transfer results are compared. A survey of the literature, however, reveals a lack of consensus with regard to what constitutes an appropriate baseline. For example, baseline performance estimates have included $50 \%$ correct (Cumming \& Berryman, 1961), greater than 50\% (Berryman, Cumming, Cohen, \& Johnson, 1965), and less than 50\% (Farthing \& Opuda, 1974). These inconsistencies are largely attributable to the use of novel transfer stimuli that frequently introduce response biases, causing experimenters to adopt various implicit and ad hoc baseline estimates. (See Sidman, 1980 , for a more complete discussion of how various types of stimulus-control factors and related response biases may produce misleading conclusions in conditional discrimination experiments.) While several "experimental" solutions to the problem have been proposed, including preexposure to novel stimuli (Farthing \& Opuda, 1974; Zentall \& Hogan, 1978) and incorporating orthogonal transfer stimuli to avoid generalization effects (Zentall \& Hogan, 1974), the present article

The research was supported by Grant A9801 from the National Research Council of Canada to Jo Tombaugh, by Grant A7074 from the Natural Sciences and Engineering Research Council of Canada to T. N. Tombaugh, and by Grant A6378 from the National Research Council of Canada to Angelo Santi. Reprints may be obtained from Jo Tombaugh, Department of Psychology, Carleton University, Ottawa, Ontario K1S 5B6, Canada. offers an alternative "statistical" procedure for assessing transfer effects that controls for possible response tendencies associated with the introduction of novel stimuli.

Assume that during the transfer phase of a matchingto-sample experiment a yellow stimulus is substituted for red in all original red-green stimulus configurations. First, percent choice to the novel stimulus is calculated, and then a difference score (percent choice to yellow when it was correct minus percent choice to yellow when it was incorrect) is computed. This score represents a measure of the relative degree to which the sample stimulus controls performance in the transfer situation, with higher scores indicating greater degrees of control. If a pigeon selected the yellow comparison $80 \%$ of the time when it served as both the sample and correct comparison and only $30 \%$ when it served as the incorrect comparison, the $50 \%$ difference score represents a high degree of sample control. On the other hand, if a pigeon selected yellow $80 \%$ of the time when it served as the sample and correct comparison and $78 \%$ of the time when it served as the incorrect comparison, the $2 \%$ difference score indicates little sample control. In the latter case, performance is controlled by an unconditional preference for the yellow stimulus. In the "high" and "low" sample-control examples, the novel stimulus was included as both the correct comparison for one stimulus pair and the incorrect comparison for the other stimulus pair. Consequently, any potential biases associated with the introduction of the novel stimulus would not be expected to differentially affect the results. The potential usefulness of the statistical treat- 
ment, suggested by the preceding analysis, was assessed in the present experiment, in which a "novel" (less familiar) stimulus was used as the transfer stimulus.

\section{METHOD}

\section{Subjects}

Four naive male White Carneaux pigeons (Palmetto Pigeon Plant) were maintained at $80 \%$ of their free-feeding body weights throughout the experiment. All animals were individually housed.

\section{Apparatus}

Four Lehigh Valley operant chambers were used. Each was enclosed in a plywood box insulated with acoustical ceiling tile. Masking noise and ventilation were provided by two 100-CFM Dayton blowers. Three translucent (Perspex) response keys, $2.54 \mathrm{~cm}$ in diameter, were arranged horizontally. The stimuli (red, green, yellow, and blue) were rear-projected on the keys by In-Line Digital Units. Directly below the center key was a $5.08-\mathrm{cm}$-square aperture giving access to a mixed-grain hopper. General illumination was provided by a houselight located above the center key. All experimental contingencies and response measures were programmed and recorded by a PDP-8/I computer located in a separate room.

\section{Procedure}

Pretraining. After the pigeons were trained to approach and eat from the hopper, an autoshaping routine was used to establish pecking to the two side keys. During autopecking, one of the side keys was illuminated. Reinforcers were presented $8 \mathrm{sec}$ following stimulus onset or immediately following a peck to the illuminated key. The order of hue presentation was randomly varied within each block of eight trials so that each individual hue was presented once on the left key and once on the right key. Throughout the experiment, daily sessions consisted of 144 trials with a $25 \mathrm{sec}$ in tertrial interval. All birds pecked consistently after seven sessions.

Chaining. The chaining phase consisted of 12 sessions. Within each session, all trials began with the illumination of the center key. A single peck to the center key illuminated one of the side keys, and a single peck to the side key resulted in the operation of the hopper mechanism for $3 \mathrm{sec}$ and the simultaneous darkening of all keys. All four hues appeared an equal number of times on the center key and each side key; the center key and illuminated side key matched on half the trials and mismatched on the other half. No food was given for pecking the center key.

Discrimination. Two birds were selected at random as matching subjects and two, as oddity subjects. Each trial began with the illumination of the center (sample) key with one of three hues: red, blue, or green. A single peck to the center key resulted in the offset of both side keys while the center key remained illuminated. A single peck to a side key resulted in the offset of the key lamps, followed by either $3 \mathrm{sec}$ of access to grain or $3 \mathrm{sec}$ of blackout, depending on whether or not the correct choice was made. Selection of the comparison stimulus that matched the center stimulus was correct for matching birds, and selection of the comparison stimulus that was different from the sample was correct for the oddity birds. Matching birds acquired the task more quickly and were given 29 sessions, with a 5-day rest during Days 26-30. Oddity birds received 34 consecutive days of training.

Transfer. Conditions during transfer were identical to those during the discrimination phase, except that a yellow light was substituted for the blue light. There were 15 transfer sessions.

\section{RESULTS AND DISCUSSION}

\section{Acquisition}

Figure 1 shows percent correct responding during acquisition and transfer for each bird (all acquisition data for Session 4 were lost due to a data processing failure). Rate of acquisition was much faster for the two matching birds than for the two oddity birds. These results are consistent with the conclusion of Carter and Werner (1978) that the rate of acquisition of a conditional discrimination is determined by the number of rules that must be learned. Thus, matching accuracy should be acquired more quickly than oddity accuracy, since there are twice the number of rules to be learned per sample stimulus in a three-key oddity paradigm (e.g., "if red, peck green" and "if red, peck blue") compared with a three-key matching situation, in which only a single rule must be learned (e.g., "if red, peck red").

\section{Transfer}

The transfer data shown in Figure 1 include responses to combinations involving the transfer stimulus (yellow), as well as to the red-green combinations that were used in acquisition. These data indicate that matching subjects attained a higher degree of accuracy than did oddity subjects. This is the same relationship observed in acquisition; however, during transfer, the lower level of asymptotic performance of the oddity birds is more evident. Apparently, the larger number of rules necessary for three-key oddity affects both rate of acquisition and asymptotic performance.

Transfer data, such as those presented in Figure 1, are typically further analyzed to identify the degree to which the various combinations of novel and familiar stimuli contribute to overall accuracy. However, the results of these analyses, based on percent accuracy scores, are critically dependent upon the type of baseline selected. This is well illustrated by the following example, in which two matching experiments obtained essentially the same effect-substantially less than 50\% accuracy for stimulus combinations involving the yellow transfer stimulus as both the sample and correct comparison

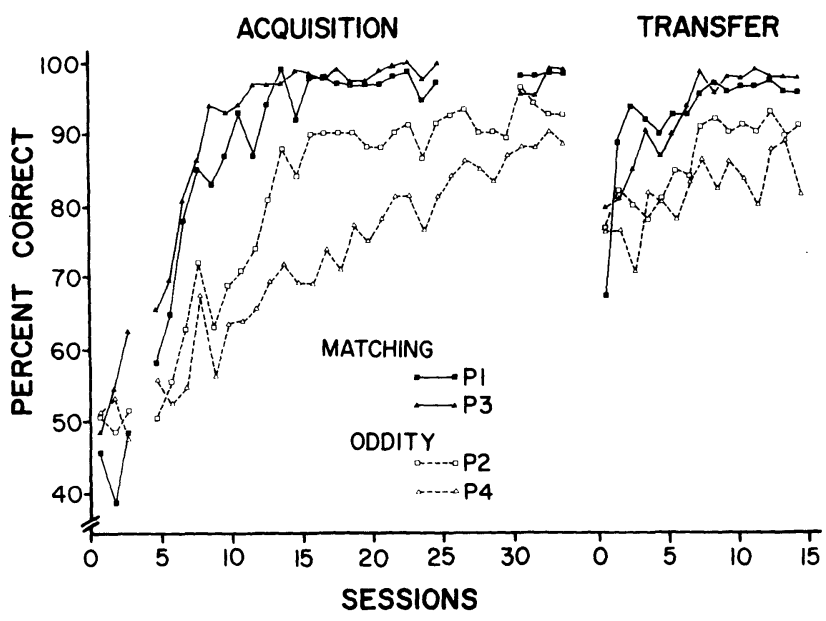

Figure 1. Percent correct for matching and oddity birds during acquisition and transfer with three hues. During training, the stimuli were red, green, and blue. In transfer, a yellow stimulus was substituted for the blue stimulus. 
Table 1

Percent Choice of Yellow (P1, P2, P3, P4) on 1st Day of Transfer for Stimulus Configurations Involving Red and Yellow and Green and Yellow

\begin{tabular}{|c|c|c|c|c|c|c|c|c|c|c|c|c|c|}
\hline \multicolumn{7}{|c|}{ Green-Yellow Configurations } & \multicolumn{7}{|c|}{ Red-Yellow Configurations } \\
\hline \multirow[b]{2}{*}{$\begin{array}{c}\text { Sample/ } \\
\text { Choices* }\end{array}$} & \multicolumn{3}{|c|}{ Matching } & \multicolumn{3}{|c|}{ Oddity } & \multirow[b]{2}{*}{$\begin{array}{c}\text { Sample/ } \\
\text { Choices* }\end{array}$} & \multicolumn{3}{|c|}{ Matching } & \multicolumn{3}{|c|}{ Oddity } \\
\hline & $\begin{array}{l}\text { Role of } \\
\text { Yellow } \dagger\end{array}$ & P1 & P3 & $\begin{array}{l}\text { Role of } \\
\text { Yellow } \dagger\end{array}$ & $\mathbf{P} 2$ & P4 & & $\begin{array}{l}\text { Role of } \\
\text { Yellow } \dagger\end{array}$ & P1 & P3 & $\begin{array}{l}\text { Role of } \\
\text { Yellow } †\end{array}$ & P2 & P4 \\
\hline G/GY & - & 0 & 25 & + & 62 & 88 & $\mathrm{R} / \mathrm{RY}$ & - & 8 & 33 & + & 54 & 38 \\
\hline Y/GY & + & 50 & 67 & - & 8 & 17 & $\mathrm{Y} / \mathrm{RY}$ & + & 29 & 42 & - & 25 & 33 \\
\hline Difference & & 50 & 42 & & 54 & 71 & Difference & & 21 & 9 & & 29 & 5 \\
\hline
\end{tabular}

Note-Difference $=$ correct minus incorrect.

${ }^{*} G=$ green $; Y=$ yellow $R=$ red. $\quad t_{-}=$incorrect $;+=$correct .

stimulus-but because of the type of baseline selected, different conclusions concerning the presence of transfer were advanced. Cumming, Berryman, and Cohen (1965) used 50\% correct responding ("chance") as the baseline and concluded that negative transfer occurred. In contrast, Farthing and Opuda (1974) argued that a baseline of less than $50 \%$ would be more appropriate, due to a tendency to avoid yellow, and consequently, they concluded that negative transfer did not occur.

Taken together, these and other studies suggest that increased attention should be directed toward determination of appropriate baselines. One way to deal with this issue is to calculate the relative degree to which the sample stimulus controls performance. Since this procedure uses identical comparison pairs in combination with various sample stimuli, any bias related to novelty will be constant over the comparison stimuli. The results obtained from this type of analysis for Day 1 of transfer are shown in Table 1, in which the various comparison stimulus combinations were first grouped into color pairs (geen-yellow, red-yellow) and then the percent choice to the novel (yellow) stimulus was calculated. (Red-green color pairs were excluded, since performance remained similar to that displayed during acquisition.) A difference score (percent choice of yellow when it was correct minus percent choice of yellow when it was incorrect) was subsequently computed for each comparison pair. This score represents a measure of relative sample control, with the higher scores indicating a greater degree of transfer. Table 1 shows that all oddity and matching birds had relatively large difference scores attributable to the sample stimulus for green-yellow combinations, but smaller difference scores for red-yellow combinations. In fact, one matching and one oddity bird showed less than a $10 \%$ difference for the red-yellow combinations, indicating almost no sample control.

The present results suggest that stimulus generalization may play a major role in determining relative sample control, with the reduced control for the redyellow combination reflecting a high degree of stimulus generalization between the comparison stimuli. This contention is supported by previous research (Wright,
1972; Wright \& Cumming, 1971) revealing a greater tendency for generalization to occur for wavelengths in the red-yellow range than for those in the yellowgreen range in paradigms such as matching to sample. When the results are viewed in this manner, no special assumptions concerning the acquisition of new "coding" responses for the sample stimuli are needed to interpret the data (see Carter \& Werner, 1978). This suggestion is underscored when the experimental data that provided the original impetus for the coding hypothesis (Berryman et al., 1965; Cumming \& Berryman, 1961) are reanalyzed by the method employed above: The same type of relative sample control is observed.

In conclusion, we have found that "relative sample control" is a convenient technique for examining conditional discrimination data when various response biases may be present. It avoids the necessity of making a priori assumptions about the manner in which possible response biases (both approach and avoidance) may influence the data. It also facilitates comparisons between matching and oddity paradigms that are differentially affected by biases toward the novel stimuli. However, although we see that "relative sample control" serves a useful function, it should not be used in lieu of experimental manipulations that empirically eliminate or control sources of response bias.

\section{REFERENCES}

Berryman, R., Cumming, W. W., Cohen, L. R., \& Johnson, D. F. Acquisition and transfer of simultaneous oddity. Psychological Reports, 1965, 17, 767-775.

Carter, D. E., \& Werner, T. J. Complex learning and information processing by pigeons: A critical analysis. Journal of the Experimental Analysis of Behavior, 1978, 29, 565-601.

Cumming, W. W., \& Berryman, R. Some data on matching behavior in the pigeon. Journal of the Experimental Analysis of Behavior, 1961, 4, 281-284.

Cumming, W. W., Berryman, R., \& Cohen, L. R. Acquisition and transfer of zero-delay matching. Psychological Reports, $1965,17,435-445$.

Farthing, G. W., \& Opuda, M. J. Transfer of matching-tosample in pigeons. Journal of the Experimental Analysis of Behavior, 1974, 21, 199-213.

Sidman, M. A note on the measurement of conditional discrim- 
ination. Journal of the Experimental Analysis of Behavior, 1980, 33, 285-290.

Wright, A. A. Psychometric and psychological hue discrimination functions for the pigeon. Vision Research, 1972, 12, 1447-1464.

Wright, A. A., \& Cumming, W. W. Color-naming functions for the pigeon. Journal of the Experimental Analysis of Behavior, 1971, 15, 7-17.

Zentall, T. R., \& Hogan, D. E. Abstract concept learning in the pigeon. Journal of Experimental Psychology, 1974, 102, 393-398.

Zentall, T. R., \& Hogan, D. E. Same/different concept learning in the pigeon: The effect of negative instances and prior adaptation to transfer stimuli. Journal of the Experimental Analysis of Behavior, 1978, 30, 177-186.

(Received for publication January 26, 1982.) 